\title{
Heart Rate Variability and Occupational Stress: Future Directions
} Newton $C^{*}$

\author{
School of Management at Queensland University of Technology, Australia
}

*Corresponding author: Newton C, School of Management at Queensland University of Technology, Australia, Fax: +61 73138 1313, Tel: +61 73138 2523, E-mail: cj.newton@qut.edu.au

Citation: Newton C (2013) Heart Rate Variability and Occupational Stress: Future Directions. J Case Rep Stud 1(1): e102. doi: 10.15744/2348-9820.1.e102

Received Date: July 07, 2013 Accepted Date: July 29, 2013 Published Date: August 01, 2013

Statistics across industrialised societies show that occupational stress results in social and financial costs for individuals, organisations, and economies. Occupational stress is prevalent in many different forms, for example, work intensification, dissatisfaction with current work schedules, feelings of job insecurity, more work being done at odd hours, the spread of new information and communication technologies, and long hours becoming more common [1]. With this work intensity comes increasing physiological and psychological strain on employees. Indeed, it has long been established that prolonged exposure to stressors incurred at work is linked to a vast array of negative attitudinal, health and, in particular, cardiovascular outcomes for employees [2].

Within the occupational stress context, the development of portable Electrocardiogram (ECG) devices provides an avenue for tracking workplace strain in a technologically advanced and minimally invasive manner. In particular, portable ECG devices provide feedback (i.e., Heart Rate Variability (HRV) biofeedback) of an individual's cardiac performance; enable individuals to witness the effects of stressful events on a minute by minute basis; and allow the direct physiological effects of stressors to be examined and subsequently targeted. However, there is still much that is unknown about the use of HRV in a stress context that requires further research. What is more, the increasing sophistication of portable measurement devices highlights the severely limited nature of much existing research through its reliance on simplistic representations of HRV.

While earlier psychological research has identified HRV as an important biological marker of stress, there has been less agreement as to the underlying mechanisms that allowed these biological systems to operate [3]. Newer research has begun to explore these pathways in greater depth, suggesting higher HRV is a marker of health by reflecting greater adaptability of the organism to manage their arousal [4]. Studies suggest higher HRV reflects higher activity in the Parasympathetic Nervous System (PNS), dominating the sympathetic nervous system (SNS). The SNS increases adrenaline and the "stress response" (i.e., increasing blood and oxygen flow to the muscles), whereas the PNS aims to regulate this stress and manage arousal [5]. This response suggests that using heart rate measures such as high frequency R-R waves (HF-HRV) reflective of parasympathetic activity is a more useful measure of the stress response compared to other heart rate measures focusing on generalized arousal commonly seen in previous research [6] and has implications for future stress research.

Another avenue for future research lies in the use of HRV biofeedback coaching as an intervention to the occupational stress process. Indeed, the search for effective stress interventions has been vast, often yielding poor or mixed results at best. Providing employees with biofeedback coaching is one way to enable them to better cope with stressors. Recent developments in wearable ECG technology have seen renewed interest in the use of HRV biofeedback for health and stress management. However, little is known about the efficacy of such interventions. What does appear relatively clear is that the process of feedback is efferent and, thus, participants in HRV programs for stress reduction require other tools and strategies to facilitate and embed new cognitions and behaviors. There are a range of other techniques including cognitive-behavioural approaches (aimed at changing cognitions and subsequently reinforcing active coping skills), relaxation and breathing techniques (focusing on physical or mental relaxation as a method to cope with the consequences of stress); and healthy lifestyle and exercise routines. In fact, it is notable that many forms of individual level stress interventions rely on reducing the impact of stressors on one's physiology, and in particular, one's cardiac physiology. However, a complexity in stress management and intervention at the individual level is related to motivating those suffering the negative effects of occupational stress to take lifestyle and intervention-related action. The most effective combination of HRV biofeedback coaching and other stress minimization techniques, in what context, and for what individual, stands out as being a key area requiring further research in the occupational stress field.

Last, but certainly not least, future research is needed to further explore the alignment of HRV/ECG with other subjective and objective indicators that can inform a greater understanding of the mechanisms underlying occupational stress and its reduction. From a subjective perspective, research questions relate the association between HRV and attitudinal outcomes such as job satisfaction, organizational commitment and 
perceptions of different stressors. Research could also further explore the alignment of HRV with personality. For example, is a stronger SNS related to greater extraversion, and PNS related to more introversion, and what are the implications for stress management? There are many research questions in this area that have yet not been fully considered by researchers. This knowledge can provide meaningful input in the quest to reduce occupational stress. From an objective perspective, researchers need to continue to explore relationships between HRV and other objective indicators of stress and strain, particularly in light of the developing knowledge of which HRV indicators are reliable and meaningful in the stress context. One such area for exploration that that stands out in particular is association with Electroencephalography (EEG). This research question is concerned with the ways that HRV/ECG indicators are related to neural patterns and brain activity. This knowledge will become increasingly useful with HRV and ECG measurement sophistication increasing in compact forms.

\section{References}

1. Sverke M, Hellgren J, Näswall K (2002) No security: A meta-analysis and review of job insecurity and its consequences. J Occup Health Psychol 7: 24264.

2. Chandola T, Brunner E, Marmot M (2006) Chronic stress at work and the metabolic syndrome: prospective study. BMJ 332: 521-5.

3. Berntson GG, Bigger JT Jr, Eckberg DL, Grossman P, Kaufmann PG, et al. (1997) Heart rate variability: origins, methods, and interpretive caveats. Psychophysiology 34: 623-48.

4. Thayer JF, Ahs F, Fredrikson M, Sollers JJ, Wager TD (2012) A meta-analysis of heart rate variability and neuroimaging studies: implications for heart rate variability as a marker of stress and health. Neurosci Biobehav Rev 36: 747-56.

5. Sharpley CF (2002) Heart rate reactivity and variability as psychophysiological links between stress, anxiety, depression, and cardiovascular disease: Implications for health psychology interventions. Australian Psychologist 37: 56-62.

6. Rozanski A, Kubzansky LD (2005) Psychologic functioning and physical health: A paradigm of flexibility. Psychosom Med 67: S47-53.

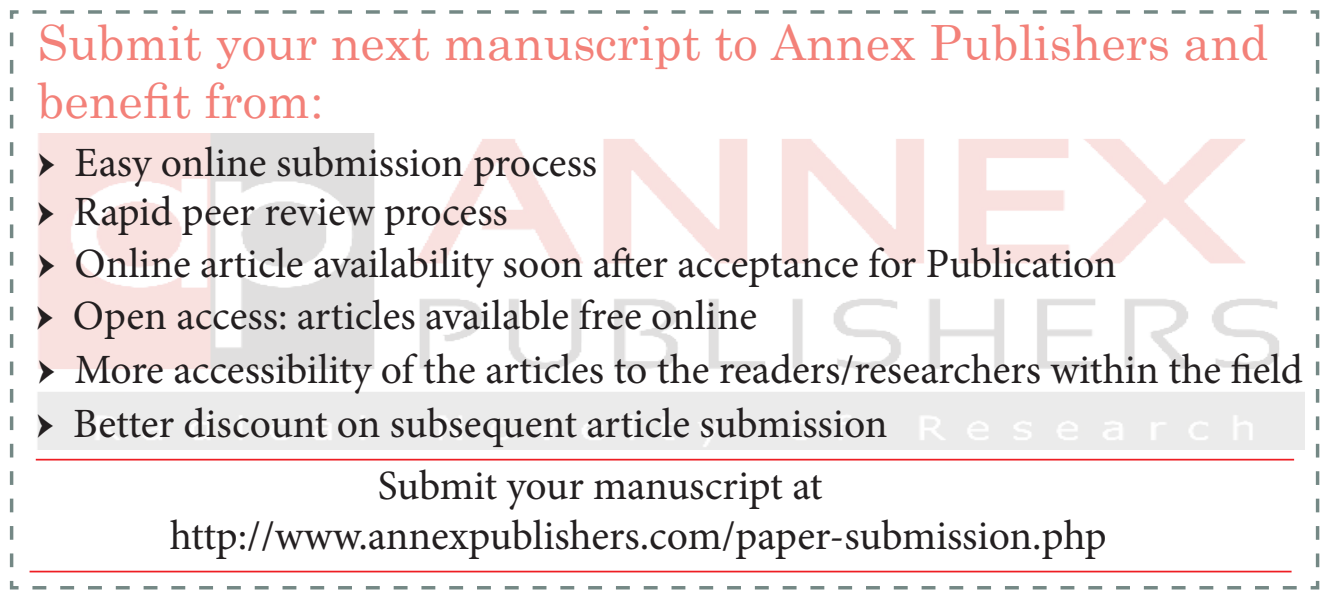

